\title{
Heart rate differences between symptomatic and asymptomatic Brugada syndrome patients at night
}

\author{
M Calvo ${ }^{1,2}$, V Le Rolle ${ }^{1}$, D Romero ${ }^{1}, \mathbf{N}$ Béhar $^{3}, \mathbf{P}$ Gomis $^{2,4}, \mathbf{P}$ \\ Mabo $^{1,3}$, A Hernández ${ }^{1}$ \\ ${ }^{1}$ LTSI - INSERM U1099, Université de Rennes 1, Rennes, 35042, France \\ 2 Dept ESAII, CREB, Universitat Politècnica de Catalunya, Barcelona, 08028, Spain \\ ${ }^{3}$ Service de Cardiologie et Maladies Vasculaires, CHU Rennes, Rennes, 35000, France \\ ${ }^{4}$ CIBER of Bioengineering, Biomaterials and Nanomedicine (CIBER-BBN), \\ Zaragoza, 50018, Spain \\ E-mail: virginie.lerolle@univ-rennes1.fr \\ March 2018
}

\begin{abstract}
Objective: Ventricular arrhythmias in Brugada syndrome (BS) mainly occur at rest, especially during nighttime, suggesting that parasympathetic activity at night may play an important role in the arrhythmogenesis of the disease. This study examined and compared the autonomic function of symptomatic and asymptomatic BS patients overnight. Approach: We analyzed different heart rate variability (HRV) and heart rate complexity (HRC) markers in a clinical series including 87 BS patients, where 23 were symptomatic. Main results: Statistically significant differences were found in MIRR, SDNN, SDANN, $\delta_{S D A N N}$ and SampEn, suggesting that symptomatic patients may be related to lower heart rate variability and complexity values, as well as to greater circadian fluctuations overnight. Significance: The results provide further evidence for the role of autonomic imbalance in the pathophysiology of BS, highlighting the relevance of nighttime analysis to unmask significant ANS changes. Based on these outcomes, the role of HRV and HRC assessment at night could be a step forward towards the understanding of BS and the risk for the occurrence of symptoms in these patients, with a potential future impact on therapeutic strategies.
\end{abstract}

Keywords: Brugada syndrome, autonomic nervous system, heart rate variability, heart rate dynamics

\section{Introduction}

Brugada syndrome (BS) is a genetic disease presenting a typical electrocardiographic pattern characterized by a distinctive ST-segment elevation in right precordial leads and a high risk of sudden cardiac death (SCD) due to ventricular fibrillation (VF) in individuals with structurally normal hearts (Priori, et al. 2013, Priori, et al. 2015). Since its first description as a distinct cardiac disease in (Brugada \& Brugada 1992), 
BS continues to spark a great research interest due to its high incidence, especially in Southeast Asia, and due to its association with SCD in young adults and, less frequently, in children and infants. The syndrome is estimated to be responsible for $4 \%$ of the total amount of SCD and for $20 \%$ of SCD in structurally normal hearts (Brugada, et al. 1998, Antzelevitch, et al. 2005).

Although several markers have been reported as predictive factors of SCD occurrence, the largest series including BS patients only determined two reliable indicators of cardiac events: prior symptoms, such as recovered SCD or syncope, and spontaneous type-1 ECG pattern (Antzelevitch et al. 2005, Probst, et al. 2010). Since implantable cardioverter defibrillators (ICD) are the only effective treatment though associated with a high incidence of adverse events (Sacher, et al. 2013), while symptomatic patients must undergo an implantation, risk stratification for asymptomatic patients still remains controversial.

Ventricular arrhythmias in BS mainly occur at rest, especially at night during sleep, suggesting that parasympathetic activity may play an important role in the arrhythmogenesis of the syndrome (Matsuo, et al. 1999, Kies, et al. 2004). Indeed, some studies on cardiac autonomic nervous system (ANS) analyzed by positron emission tomography have evidenced that BS patients display some autonomic dysfunction (Kies et al. 2004, Wichter, et al. 2002, Paul, et al. 2011, Bigi, et al. 2008). Thus, changes in the autonomic modulation assessed by heart rate variations might provide useful information about the arrhythmic risk of these patients.

Previous studies assessing the autonomic function through heart rate variability (HRV) analyses along 24-hour ECG recordings have led to contradictory results (Krittayaphong, et al. 2003, Hermida, et al. 2003, Pierre, et al. 2007, Tokuyama, et al. 2014, Nakazawa, et al. 2003, Kostopoulou, et al. 2010, Behar, et al. 2016). Moreover, since linear temporal and spectral HRV parameters are unable to capture the complexity of heart rate regulation, features measuring the nonlinear dynamics of RR series, such as Sample Entropy (SampEn), could better predict the risk of suffering symptoms in BS. Although we already reported significant results in heart rate complexity (HRC) during exercise testing in a previous work including BS patients (Calvo, et al. 2017), we are not aware of any study having assessed HRC at night.

Therefore, in order to identify significant differences in the autonomic function of BS patients at different levels of risk (symptomatic and asymptomatic subjects), the aim of this study was to compare HRV and HRC markers and its circadian fluctuations at night in a clinical series including $87 \mathrm{BS}$ patients. The evaluation of heart rate variability and complexity markers may provide supplementary information that could be useful for risk stratification in asymptomatic BS patients. 


\section{Methods}

\subsection{Study population}

The 24-hour continuous ECG recordings of 87 patients diagnosed with Brugada syndrome were collected in the context of a prospective, multicentric study, conducted between 2009 and 2013 in the Cardiology department of the Rennes University Hospital, in France. Participants were enrolled in 8 French hospitals located in Rennes, Tours, Poitiers, Angers, Nantes, Bordeaux, Brest and La Rochelle. The protocol was approved by the respective local ethics committees before the study recruitment began in 2009, and all patients provided written informed consent before participation.

In accordance with the current guidelines (Priori et al. 2015, Priori et al. 2013), BS was diagnosed when a coved ST-segment elevation $(\geq 0.2 \mathrm{mV})$ was recorded in at least one right precordial lead (V1 and/or V2) located in the $2^{\text {nd }}, 3^{\text {rd }}$ or $4^{\text {th }}$ intercostal space, in the presence or absence of sodium-channel-blocking agent.

Participant ages ranged from 19 to 79 years old $(45.63 \pm 13.03$ years old) and $71.26 \%$ were men. Based on their medical history, they were classified as symptomatic and asymptomatic. Twenty-three patients had the following documented symptoms of ventricular origin: syncope $(60.87 \%)$, cardiac arrest $(34.78 \%)$, dizziness $(13.04 \%)$ and, less frequently, palpitations $(4.35 \%)$ and nocturnal convulsions $(4.35 \%)$. The remaining 64 patients were considered as asymptomatic.

Cardioverter defibrillator implantation had been performed in 15 of $64(23.44 \%)$ asymptomatic patients, based on a positive EPS (Electrophysiological Study) test, whereas all symptomatic patients were ICD carriers. Since no relevant cardiac events were noted at night, defibrillators caused no significant effects on the recordings.

Genetic testing in search of SCN5A mutations was completed in 71 patients (21 were symptomatic) and is pending in 6 asymptomatic and 1 symptomatic subjects. The test was not performed in 9 patients (1 symptomatic), as no mutation had been identified in their families. Among 27 patients in whom the SCN5A mutation was found, 10 were symptomatic.

Table 1 summarizes the clinical characteristics of patients included in the study. Since no significant differences in age, gender and SCN5A-mutation presence between symptomatic and asymptomatic groups was noted ( $p$-value $>0.05)$, similar baseline characteristics were assumed between populations.

\subsection{Signal acquisition and preprocessing}

The standard 12-lead ECG recordings of each patient were acquired with the Holter monitor (ELA medical, Sorin Group, Le Plessis Robinsson, France), at a sampling frequency of $1000 \mathrm{~Hz}$. For this study, only the period from midnight to 6 a.m. was evaluated, so as to make sure that autonomic analysis was performed while patients were asleep.

RR series were obtained using a noise-robust wavelet-based algorithm for QRS 
Heart rate differences in Brugada syndrome at night

Table 1. Clinical characteristics of asymptomatic and symptomatic study groups.

\begin{tabular}{lccc}
\hline & $\begin{array}{c}\text { Asymptomatic } \\
(\mathbf{n = 6 4 )}\end{array}$ & $\begin{array}{c}\text { Symptomatic } \\
(\mathbf{n = 2 3})\end{array}$ & $p$-value \\
\hline Age, years old & $45.50 \pm 13.08$ & $46.00 \pm 13.16$ & 0.996 \\
Male sex, $n(\%)$ & $43(67.19 \%)$ & $19(82.61 \%)$ & 0.163 \\
ICD implantation, $n(\%)$ & $15(23.44 \%)$ & $23(100 \%)$ & - \\
Cardiac arrest, $n(\%)$ & - & $8(30.43 \%)$ & - \\
Syncope, $n(\%)$ & - & $14(60.87 \%)$ & - \\
Near syncope/dizziness, $n(\%)$ & - & $3(13.04 \%)$ & - \\
Palpitations, $n(\%)$ & - & $1(4.35 \%)$ & - \\
Nocturnal convulsions, $n(\%)$ & - & $1(4.35 \%)$ & - \\
Presence of SCN5A mutation, $n(\%)$ & $17(34.00 \%)$ & $10(47.62 \%)$ & 0.284 \\
\hline
\end{tabular}

complex detection and subsequent R-wave peak location (Dumont, et al. 2010). So as to minimize the effect of artifacts and ectopic beats, RR series were filtered using a 5-beat sliding window algorithm that rejects those beats deviating more than $15 \%$ from the average length of the preceding intervals (Clifford, et al. 2002). Rejected beats were then replaced by the previous 5-beat RR mean (Wessel, et al. 2000), and the quality of $\mathrm{RR}$ series was finally verified by visual inspection.

For frequency analysis, since RR series were not uniformly sampled with respect to time, a cubic splines interpolation was applied to data, in order to obtain regularlysampled series, at a rate of $4 \mathrm{~Hz}$. Moreover, so as to remove DC and very low frequency components, RR series were high-pass filtered at $0.03 \mathrm{~Hz}$, with a $4^{\text {th }}$ order Butterworth filter applied in both forward and backward directions.

\subsection{Heart rate variability}

According to the Task Force on HRV (Camm, et al. 1996), the following time-domain variables were computed: mean and standard deviation of $\mathrm{RR}$ intervals $(\overline{R R}$ and $S D N N), N N 50$ as the number of pairs of adjacent RR intervals differing by more than 50 milliseconds and $p N N 50$ as the proportion of $N N 50$ divided by the total number of intervals. Additionally, $M I R R$ was calculated as the interquartile range of the RR sequence, $S D A N N$ as the standard deviation of the entire 5-minute RR means, SDNN5min as the mean of the 5-minute RR standard deviations and $r M S S D$ as the root mean square of the successive difference between adjacent $R R$ intervals.

Parametric modeling analysis such as the autoregressive (AR) model using the Burg method provides high-resolution spectral estimation of short-term signals as the RR windowed segments studied in (Burg 1975). The estimation is based on the minimization of the forward and backward prediction errors using the least squares method. The AR model order was optimized for each signal, using the Broersen's combined information criterion (Broersen 2000), in order to extract the spectral HRV indices from the resulting 
Power Spectral Density (PSD).

The total power (TP) was obtained as the sum of the four spectral bands: ultra-low frequency (ULF: 0-0.003 Hz), very-low frequency (VLF: 0.003-0.04 Hz), low frequency (LF: 0.04-0.15 Hz) and high frequency (HF: 0.15-0.4 Hz). The HF component is defined as a marker of vagal modulation and the LF component is modulated by both the sympathetic and parasympathetic nervous systems, leading to controversial interpretations (Camm et al. 1996). These were calculated from the average of 5-minute intervals and normalized by subtracting the VLF component from the total power in order to reduce the effects of noise artifacts:

$$
\begin{aligned}
& L F_{n u}=\frac{L F}{T P-V L F} \\
& H F_{n u}=\frac{H F}{T P-V L F}
\end{aligned}
$$

The $\mathrm{LF} / \mathrm{HF}$ ratio reflects the global sympathovagal balance and can be used as a measure of this balance.

\subsection{Heart rate complexity}

According to a critical review of newly developed HRV methods published after the Task Force on HRV (Sassi, et al. 2015), although several nonlinear markers, such as those capturing the chaotic behavior of HRV, have been proposed to characterize HRC, the results collected thus far are still too limited to conclude that human HRV arises from a chaotic behavior of the cardiovascular system.

Conversely, according to the same review, entropy measures such as sample entropy (SampEn) seem to accurately represent the responsiveness of the cardiovascular system to environmental stimuli, decreasing when RR series become more ordered and predictable. Therefore, HRC was herein evaluated through the analysis of the entropy captured by SampEn. This marker is indeed a refined version of the traditionally used irregularity measure Approximate Entropy ( $A p E n$ ) (Richman \& Moorman 2000). It is defined as the conditional probability that two sequences of $m$ consecutive data points which are similar to each other (with a tolerance $r$ ) will remain similar when one more consecutive point is included. Large values of SampEn indicate high irregularity and great complexity, whereas smaller values indicate a more regular and predictable signal, commonly associated with disease (Khandoker, et al. 2009, Javorka, et al. 2008, Tuzcu, et al. 2006).

Although no consensus has been yet established for the appropriate selection of the embedding dimension $m$ and tolerance threshold $r$, based on the exhaustive evaluation performed by (Lake, et al. 2002) that led to the selection of $m=3$ and $r=0.2 \cdot S D$, we chose, as in (Kim, et al. 2005, Tuzcu et al. 2006), this combination of parameters. 


\subsection{Statistical analysis}

Kolmogorov-Smirnov tests were applied so as to assess normality on extracted markers. Since, according to the results, normal distributions could not be assumed, statistical comparisons between symptomatic and asymptomatic patients during the whole night (midnight to 6 a.m.), as well as for each interval of one hour, were evaluated by Mann-Whitney U non-parametric tests. Moreover, fluctuations in HRV at night were calculated as in (Behar et al. 2016), using a coefficient of variation $\delta=$ (maximum minimum value)/maximum value.

Logistic regression is a statistical method that finds the best fitting model to describe the relationship between a dichotomous outcome (here the presence/absence of symptoms) and a set of independent variables $X_{1}, \cdots, X_{k}$. It generates the coefficients $b_{o}, b_{1}, \cdots, b_{k}$ (and its standard errors and significance levels) that maximize the likelihood of observing the sample values in order to predict a logit transformation of the probability of suffering symptoms from the following formula:

$$
\operatorname{logit}(p)=\ln \frac{p}{1-p}=b_{0}+b_{1} X_{1}+\cdots+b_{k} X_{k}
$$

Therefore, binary logistic regression models were applied to each variable differing significantly between groups in order to predict the probability of experiencing symptoms related to those HRV and HRC measures. By calculating the odds ratios (OR) related to these models, we quantified how strongly the presence of each extracted feature may be associated with the presence of symptoms in Brugada syndrome.

HRV parameter calculations and statistical tests were performed by applying algorithms developed in our laboratory for HRV analysis, using the commercially available software MatLab (Mathworks Inc., MI, USA) and setting the level of significance at $p<0.05$.

\section{Results}

When the whole night was taken into account, similar results with no significant differences between study groups were found. However, the variation coefficient of $S D A N N, \delta S D A N N$, was significantly higher in symptomatic than in asymptomatic patients between 1 a.m. and 5 a.m. $(p<0.005)$.

Regarding heart rate variability and complexity differences analyzed for each interval of one hour, some relevant tendencies, represented by some exemplifying markers in Figure 1, were observed. Table 2 summarizes the mean \pm standard deviation values for symptomatic and asymptomatic patients and the associated $p$-values obtained in each significant case. Results from all the analyzed markers are provided as Supplementary material.

Symptomatic patients showed a decreased heart rate variability, which was statistically significant between 5 a.m. - 6 a.m., according to $S D N N, M I R R$ and $S D A N N$. Significant differences were also captured by $M I R R$ between midnight - 1 
Heart rate differences in Brugada syndrome at night

Table 2. Mean \pm standard deviation, for asymptomatic and symptomatic patients, and associated $p$-values of significant variables.

\begin{tabular}{|c|c|c|c|}
\hline & $\begin{array}{l}\text { Asymptomatic } \\
(\mathrm{n}=64)\end{array}$ & $\begin{array}{l}\text { Symptomatic } \\
\quad(n=23)\end{array}$ & $p$-value \\
\hline \multicolumn{4}{|l|}{$S D N N$} \\
\hline $\begin{array}{l}\quad \mathbf{5} \text { a.m. }-\mathbf{6} \text { a. } \mathbf{m} . \\
M I R R\end{array}$ & $97.04 \pm 36.93$ & $79.48 \pm 33.65$ & 0.029 \\
\hline Midnight - 1 a.m. & $88.95 \pm 59.16$ & $64.48 \pm 29.10$ & 0.032 \\
\hline 5 a.m. - 6 a.m. & $113.86 \pm 62.46$ & $85.74 \pm 49.64$ & 0.014 \\
\hline \multicolumn{4}{|l|}{$S D A N N$} \\
\hline 2 a.m. - 3 a.m. & $38.77 \pm 23.81$ & $26.83 \pm 16.16$ & 0.027 \\
\hline 5 a.m. -6 a.m. & $46.65 \pm 24.59$ & $35.16 \pm 22.90$ & 0.025 \\
\hline$\delta_{S D A N N}$ (1 a.m. - 5 a.m.) & $0.56 \pm 0.14$ & $0.67 \pm 0.10$ & $<0.005$ \\
\hline \multicolumn{4}{|l|}{ SampEn } \\
\hline 1 a.m. - 2 a.m. & $1.19 \pm 0.25$ & $1.04 \pm 0.36$ & 0.045 \\
\hline 4 a.m. - 5 a.m. & $1.12 \pm 0.26$ & $0.95 \pm 0.37$ & 0.017 \\
\hline
\end{tabular}

a.m. and by $S D A N N$ between 2 a.m. - 3 a.m. Similarly, they displayed reduced values for SampEn with respect to asymptomatic subjects, which turned to be significant between 1 a.m. - 2 a.m. and 4 a.m. - 5 a.m.

Table 3 summarizes the odds ratios (OR), 95\% confidence intervals (CI) and $p$ values associated to each univariate model. In summary, MIRR between midnight - 1 a.m. turned to be an independent predictor for the occurrence of symptoms in univariate analysis with a $1.7 \%$ decreased risk per one point increase. Between 2 a.m. - 3 a.m., $S D A N N$ displayed a decreased risk of the 3.1\%. $\delta S D A N N$ between 1 a.m. - 5 a.m. was also a significant predictor of symptoms, as well as SampEn between 1 a.m. - 2 a.m. and between 4 a.m. - 5 a.m. showed a decreased risk of the $82.9 \%$ and $87.1 \%$, respectively, per one point of SampEn increase.

Figure 1 represents the circadian variation of $\mathrm{HRV}$ and $\mathrm{HRC}$ at night for both groups of patients, by showing the evolution of the mean, and the $25 \%$ of the standard deviation, at each hour for the most significant autonomic markers. Likewise, the main tendency of SampEn and MIRR is represented by fitting linear regression lines for each group of patients. The graphs show a similar evolution along the night in terms of regression line slopes between both groups, but symptomatic patients display lower mean heart rate variability and complexity values.

\section{Discussion}

In this study, the autonomic function of $87 \mathrm{BS}$ patients was analyzed at night through several HRV and HRC markers. Although some previous studies have already assessed HRV on these patients, this manuscript presents a more extensive clinical series than those reported thus far and proposes the first comparison of HRC between symptomatic 
Table 3. Odds ratios, $95 \%$ confidence intervals and $p$-values from univariate analysis of significant autonomic markers.

\begin{tabular}{|c|c|c|c|}
\hline & OR & $95 \% \mathrm{CI}$ & $p$-value \\
\hline \multicolumn{4}{|l|}{$S D N N$} \\
\hline 5 a.m. -6 a. m. & 0.984 & $0.969-1.000$ & 0.053 \\
\hline \multicolumn{4}{|l|}{$M I R R$} \\
\hline Midnight - 1 a.m. & 0.983 & $0.967-1.000$ & $0.047^{*}$ \\
\hline 5 a.m. - 6 a.m. & 0.989 & $0.978-1.000$ & 0.060 \\
\hline \multicolumn{4}{|l|}{$S D A N N$} \\
\hline 2 a.m. -3 a.m. & 0.969 & $0.941-0.998$ & $0.037^{*}$ \\
\hline 5 a.m. - 6 a.m. & 0.978 & $0.956-1.001$ & 0.059 \\
\hline$\delta_{S D A N N}$ (1 a.m. - 5 a.m.) & 1039.321 & $11.293-95650.848$ & $0.003^{*}$ \\
\hline \multicolumn{4}{|l|}{ SampEn } \\
\hline 1 a.m. - 2 a.m. & 0.171 & $0.032-0.913$ & $0.039^{*}$ \\
\hline 4 a.m. - 5 a.m. & 0.129 & $0.023-0.714$ & $0.019^{*}$ \\
\hline
\end{tabular}

${ }^{*} p<0.05$ when comparing symptomatic and asymptomatic study groups.

Figure 1. Mean and $25 \%$ of standard deviation, for SampEn and MIRR on symptomatic and asymptomatic BS patients at each hour during nighttime $\left({ }^{*} p<0.05\right)$. Dotted black lines are fitted linear regression lines that represent the main tendencies of each variable along the night, for both study groups.
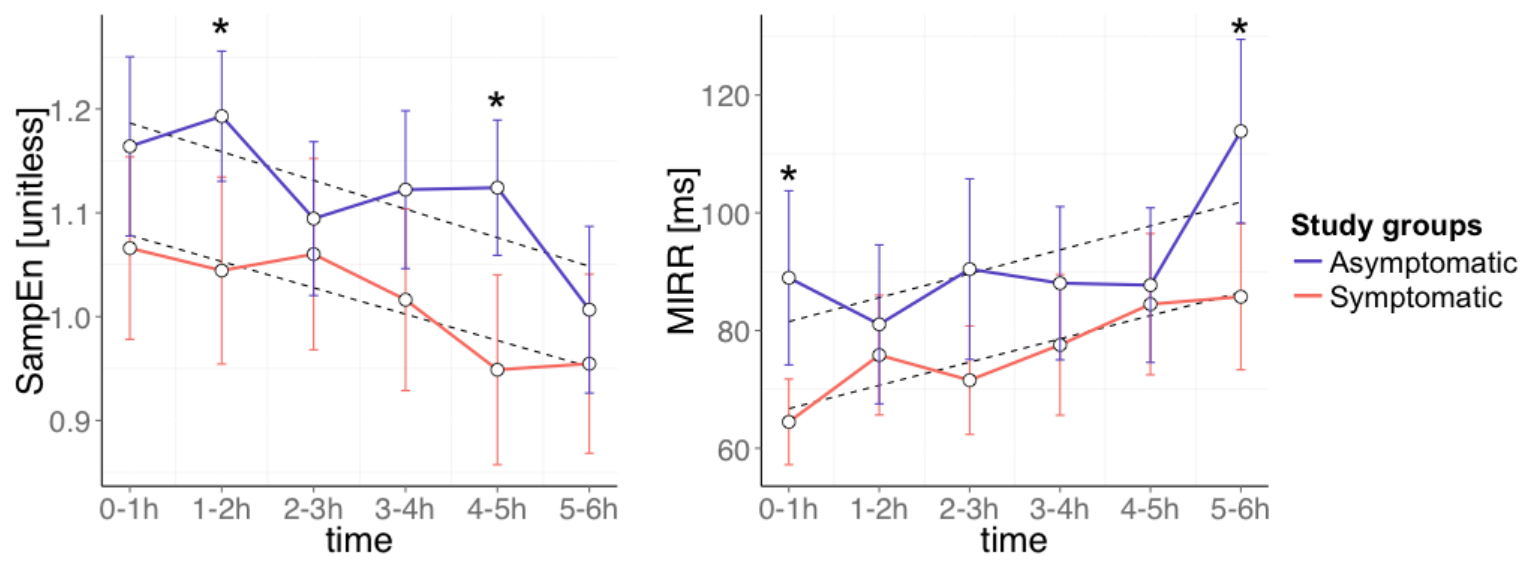

and asymptomatic BS patients overnight.

In a clinical series of 27 patients with BS, of whom 10 were asymptomatic subjects with Brugada ECG, and 45 controls, Krittayaphong et al (Krittayaphong et al. 2003) found that symptomatic BS patients showed lower HRV and lower vagal tone at night compared to controls, as well as lower diurnal and higher overnight heart rates compared to asymptomatic subjects and controls. Similarly, Hermida et al (Hermida et al. 2003) detected a significant reduction in $S D A N N$ at night when comparing Holter recordings in 21 symptomatic and 26 asymptomatic BS patients. Pierre et al (Pierre et al. 2007) 
also confirmed in a clinical series of 46 BS patients and 46 controls that HRV in BS was significantly lower with respect to healthy subjects. Tokuyama et al (Tokuyama et al. 2014) results showed a significant reduction of the LF and HF components in BS patients with previous VF when analyzing a series of 12 symptomatic (with previous VF), 17 asymptomatic and 16 healthy individuals. The results showed a decreased circadian variation of the autonomic response over 24 hours, with respect to controls. Conversely, Nakazawa et al (Nakazawa et al. 2003) analyzed, in 24-hour ECG recordings, the autonomic function of $27 \mathrm{BS}$ patients (10 of them had a history of VF and 17 did not) and of 26 healthy subjects. They found higher vagal and reduced sympathetic activities (increase of HF component and decrease of LF/HF) in BS patients with previous VF compared to controls, as well as an increase of HF component when compared to asymptomatic BS patients. Kostopoulou et al (Kostopoulou et al. 2010) did not find any significant differences in HRV between 20 patients with BS and 20 age-matched controls, although a high susceptibility to vasovagal syncope during head-up tilt test in BS patients was noticed.

In our study, according to time-domain HRV markers, symptomatic patients showed lower MIRR, SDNN and $S D A N N$ values, mainly during the last hour (5 a.m. - 6 a.m.). Therefore, patients with higher risk for cardiac events seem to show a decreased HRV, following the tendency published in previous works (Krittayaphong et al. 2003, Hermida et al. 2003, Pierre et al. 2007, Tokuyama et al. 2014), emphasizing differences during the last hour of sleep.

From HRC analysis, statistically significant results were observed between 2 a.m. - 3 a.m. and 4 a.m. - 5 a.m. Symptomatic patients showed more regularity in RR series, which might be related to a worse cardiac health condition since a reduced complexity in HR has been associated with disease (Khandoker et al. 2009, Javorka et al. 2008, Tuzcu et al. 2006). The ANS is controlled by complex interactions resulting from feedback loops of nonlinear systems that permit the organism to adapt to particular physiological conditions, such as metabolic variations, stress or disease. Thus, according to previous studies, a reduction in the complexity of biological control systems reflects a decreased organism adaptability, generally associated with age and disease (Goldberger, et al. 2002, Rabinovich \& Abarbanel 1998, Magrans, et al. 2010, Smith, et al. 2005).

In a previous study, we reported reduced values of $\mathrm{HRC}$ in symptomatic BS patients with respect to asymptomatic subjects, during recovery after physical stress testing (Calvo et al. 2017). As it was suggested, this affected ANS dynamics when parasympathetic activity is predominant confirms previous studies that highlight the importance of autonomic imbalance in the genesis of life-threatening arrhythmias in BS (Matsuo et al. 1999, Kies et al. 2004). Indeed, ventricular arrhythmias and sudden cardiac death (SCD) mainly occur at rest and especially during sleep, when vagal tone is dominant.

Regarding circadian fluctuations, based on $\delta_{S D A N N}$ results, we observed significantly greater variations during nighttime in symptomatic than in asymptomatic patients, which concurs with previous studies having compared these groups during 
daytime and when the night was analyzed between midnight and 4 a.m. As suggested by (Behar et al. 2016), this increase in $\delta_{S D A N N}$ might be caused by wider fluctuations in the autonomic tone, or by a greater sinus node autonomic receptors sensitivity.

Then, we included those statistically significant variables in univariate logistic regression models in order to estimate the discrimination power of each parameter. According to HRV results, MIRR turned to be an independent predictor between midnight and 1 a.m.; $S D A N N$ at 2 a.m. - 3 a.m; and $\delta_{S D A N N}$ between 1 a.m. and 5 a.m. HRC captured by $S a m p E n$ was also a significant predictor for the occurrence of cardiac events.

Thus, our findings provide further evidence for the role of autonomic imbalance in the pathophysiology of the Brugada syndrome. Although we did not find significant differences between symptomatic and asymptomatic groups in HRV markers characterizing the HF component that would directly relate the loss of cardiac variability and complexity with an increase in parasympathetic activity, the results highlight the relevance of night analysis in order to unmask significant autonomic changes in symptomatic BS patients.

These results support the idea that decreased nocturnal HR variability and complexity, as well as a greater circadian variation, could be related to a greater risk of suffering symptoms in Brugada syndrome. Measuring HRV not only by conventional but also by nonlinear methods could be a helpful tool to better understand autonomic alterations in BS, with a potential impact on risk stratification and, thus, on therapeutic strategies.

Although cardioverter defibrillators are the only proven effective treatment thus far, since they are associated with a high incidence of adverse events, the decision to implant them on asymptomatic subjects is still contentious, even if they represent around the $60 \%$ of diagnosed patients. Thus, the proposed indices are presented as a potential instrument to better identify those asymptomatic BS patients at high risk who may benefit from an ICD implantation. Moreover, they might also be applied to data acquired from ICDs on already implanted BS patients, in order to control their risk of suffering symptoms during follow-up.

\section{Conclusions}

In this study, we analyzed and compared several HRV and HRC markers in 87 subjects in order to identify autonomic differences between symptomatic and asymptomatic BS patients at night. Statistically significant differences were observed in several temporal $\left(M I R R, p=0.014 ; S D N N, p=0.029 ; \delta_{S D A N N}, p<0.005 ; S D A N N, p=0.025\right)$ and nonlinear ( $S a m p E n, p=0.017$ ) markers, especially during the last period of sleep. The results suggest that symptomatic patients may be related to lower heart rate variability and complexity values, as well as to greater circadian fluctuations overnight.

The study presents some limitations that should be noted. First, the clinical influence of the analyzed markers can only be proved if a direct relationship between 
heart rate alterations and cardiac events is identified. Here, symptomatic patients had documented symptoms but since no ventricular arrhythmias were induced during the recordings, heart rate variations in symptomatic patients cannot be directly associated to a greater risk for suffering symptoms. Moreover, the analysis is based on a relatively small population of 87 patients and, thus, conclusions on predictors of symptoms cannot be extracted. Finally, although differences in the autonomic function of different sleep stages have been reported, since accurate sleep-phase identifications require electroencephalogram, electrooculogram and electromyogram data not available for this study, the obtained results may not be a reliable measure of the autonomic function at night.

Nevertheless, the observed differences between symptomatic and asymptomatic BS patients indicate important trends of clinical relevance that could provide new insights in the understanding of the physiopathology of the syndrome. Future work will be based on combining these parameters, together with other relevant markers of risk, on a multivariate classification method capable of identifying BS patients who may benefit from an ICD implantation.

\section{Acknowledgments}

This work was supported by the French Ministry of Health (Programme Hospitalier de Recherche Clinique - PHRC Regional). MC thanks la Caixa Foundation (Spain) and DR acknowledges Lefoulon-Delalande Foundation (France) for financial support.

\section{References}

C. Antzelevitch, et al. (2005). 'Brugada Syndrome: Report of the Second Consensus Conference: Endorsed by the Heart Rhythm Society and the European Heart Rhythm Association'. Circulation 111(5):659-670.

N. Behar, B. Petit, V. Probst, F. Sacher, G. Kervio, J. Mansourati, P. Bru, A. Hernandez \& P. Mabo (2016). 'Heart rate variability and repolarization characteristics in symptomatic and asymptomatic Brugada syndrome'. Europace p. euw224.

M. A. B. Bigi, A. Aslani \& A. Aslani (2008). 'Significance of cardiac autonomic neuropathy in risk stratification of Brugada syndrome'. Europace 10(7):821-824.

P. Broersen (2000). 'Finite sample criteria for autoregressive order selection'. Signal Processing, IEEE Transactions on $48(12): 3550-3558$.

J. Brugada, R. Brugada \& P. Brugada (1998). 'Right Bundle-Branch Block and ST-Segment Elevation in Leads V1 Through V3 : A Marker for Sudden Death in Patients Without Demonstrable Structural Heart Disease'. Circulation 97(5):457-460.

P. Brugada \& J. Brugada (1992). 'Right bundle branch block, persistent \{ST\} segment elevation and sudden cardiac death: A distinct clinical and electrocardiographic syndrome: A multicenter report'. Journal of the American College of Cardiology 20(6):1391 - 1396.

J. P. Burg (1975). Maximum Entropy Spectral Analysis. Phd thesis, Stanford University, Stanford, CA 94305.

M. Calvo, P. Gomis, D. Romero, V. Le Rolle, N. Béhar, P. Mabo \& A. Hernández (2017). 'Heart rate complexity analysis in Brugada syndrome during physical stress testing'. Physiological measurement 38(2):387. 
A. Camm, et al. (1996). 'Heart rate variability: standards of measurement, physiological interpretation and clinical use. Task Force of the European Society of Cardiology and the North American Society of Pacing and Electrophysiology'. Circulation 93(5):1043-1065.

G. Clifford, P. McSharry \& L. Tarassenko (2002). 'Characterizing artefact in the normal human 24hour RR time series to aid identification and artificial replication of circadian variations in human beat to beat heart rate using a simple threshold'. In Computers in Cardiology, 2002, pp. 129-132. IEEE.

J. Dumont, A. Hernandez \& G. Carrault (2010). 'Improving ECG Beats Delineation With an Evolutionary Optimization Process'. Biomedical Engineering, IEEE Transactions on 57(3):607615.

A. L. Goldberger, C.-K. Peng \& L. A. Lipsitz (2002). 'What is physiologic complexity and how does it change with aging and disease?'. Neurobiology of aging 23(1):23-26.

J.-S. Hermida, A. Leenhardt, B. Cauchemez, I. Denjoy, G. Jarry, F. Mizon, P. Milliez, J.-L. Rey, P. Beaufils \& P. Coumel (2003). 'Decreased nocturnal standard deviation of averaged NN intervals'. European heart journal 24(22):2061-2069.

M. Javorka, Z. Trunkvalterova, I. Tonhajzerova, J. Javorkova, K. Javorka \& M. Baumert (2008). 'Short-term heart rate complexity is reduced in patients with type 1 diabetes mellitus'. Clinical Neurophysiology 119(5):1071-1081.

A. H. Khandoker, H. F. Jelinek \& M. Palaniswami (2009). 'Identifying diabetic patients with cardiac autonomic neuropathy by heart rate complexity analysis'. Biomedical engineering online 8(1):3.

P. Kies, et al. (2004). 'Abnormal Myocardial Presynaptic Norepinephrine Recycling in Patients With Brugada Syndrome'. Circulation 110(19):3017-3022.

W.-S. Kim, Y.-Z. Yoon, J.-H. Bae \& K.-S. Soh (2005). 'Nonlinear characteristics of heart rate time series: influence of three recumbent positions in patients with mild or severe coronary artery disease'. Physiological measurement 26(4):517.

A. Kostopoulou, et al. (2010). 'Disorders of the Autonomic Nervous System in Patients With Brugada Syndrome: A Pilot Study'. Journal of Cardiovascular Electrophysiology 21(7):773-780.

R. Krittayaphong, G. Veerakul, K. Nademanee \& C. a. Kangkagate (2003). 'Heart rate variability in patients with Brugada syndrome in Thailand'. European Heart Journal 24(19):1771-1778.

D. E. Lake, J. S. Richman, M. P. Griffin \& J. R. Moorman (2002). 'Sample entropy analysis of neonatal heart rate variability'. American Journal of Physiology-Regulatory, Integrative and Comparative Physiology 283(3):R789-R797.

R. Magrans, P. Gomis, P. Caminal \& G. Wagner (2010). 'Multifractal and nonlinear assessment of autonomous nervous system response during transient myocardial ischaemia'. Physiological measurement 31(4):565-580.

K. Matsuo, T. Kurita, M. Inagaki, M. Kakishita, N. Aihara, W. Shimizu, A. Taguchi, K. Suyama, S. Kamakura \& K. a. Shimomura (1999). 'The circadian pattern of the development of ventricular fibrillation in patients with Brugada syndrome'. European Heart Journal 20(6):465-470.

K. Nakazawa, T. Sakurai, A. Takagi, R. Kishi, K. Osada, T. Nanke, F. Miyake, N. Matsumoto \& S. Kobayashi (2003). 'Autonomic imbalance as a property of symptomatic Brugada syndrome'. Circulation journal : official journal of the Japanese Circulation Society 67(6):511-514.

M. Paul, M. Meyborg, P. Boknik, U. Gergs, W. Schmitz, G. Breithardt, T. Wichter \& N. Joachim (2011). 'Autonomic Dysfunction in Patients with Brugada Syndrome: Further Biochemical Evidence of Altered Signaling Pathways'. Pacing and Clinical Electrophysiology 34(9):1147-1153.

B. Pierre, D. Babuty, P. Poret, C. Giraudeau, O. Marie, P. Cosnay \& L. Fauchier (2007). 'Abnormal nocturnal heart rate variability and QT dynamics in patients with Brugada syndrome'. Pacing and clinical electrophysiology 30(s1):S188-S191.

S. G. Priori, et al. (2015). '2015 ESC Guidelines for the management of patients with ventricular arrhythmias and the prevention of sudden cardiac death: The Task Force for the Management of Patients with Ventricular Arrhythmias and the Prevention of Sudden Cardiac Death of the European Society of Cardiology (ESC) Endorsed by: Association for European Paediatric and 
Congenital Cardiology (AEPC)'. European heart journal 36(41):2793-2867.

S. G. Priori, et al. (2013). 'Executive summary: HRS/EHRA/APHRS expert consensus statement on the diagnosis and management of patients with inherited primary arrhythmia syndromes'. Europace 15(10):1389-1406.

V. Probst, et al. (2010). 'Long-term prognosis of patients diagnosed with Brugada syndrome results from the FINGER Brugada Syndrome Registry'. Circulation 121(5):635-643.

M. Rabinovich \& H. Abarbanel (1998). 'The role of chaos in neural systems'. Neuroscience 87(1):5-14.

J. S. Richman \& J. R. Moorman (2000). 'Physiological time-series analysis using approximate entropy and sample entropy'. American Journal of Physiology-Heart and Circulatory Physiology 278(6):H2039-H2049.

F. Sacher, et al. (2013). 'Outcome after implantation of a cardioverter-defibrillator in patients with Brugada syndrome'. Circulation 128(16):1739-1747.

R. Sassi, et al. (2015). 'Advances in heart rate variability signal analysis: joint position statement by the e-Cardiology ESC Working Group and the European Heart Rhythm Association co-endorsed by the Asia Pacific Heart Rhythm Society'. EP Europace 17(9):1341-1353.

R. G. Smith, L. Betancourt \& Y. Sun (2005). 'Molecular endocrinology and physiology of the aging central nervous system'. Endocrine reviews 26(2):203-250.

T. Tokuyama, et al. (2014). 'Deterioration of the circadian variation of heart rate variability in Brugada syndrome may contribute to the pathogenesis of ventricular fibrillation'. Journal of cardiology 64(2):133-138.

V. Tuzcu, S. Nas, T. Börklü \& A. Ugur (2006). 'Decrease in the heart rate complexity prior to the onset of atrial fibrillation'. Europace 8(6):398-402.

N. Wessel, A. Voss, J. Kurths, A. Schirdewan, K. Hnatkova \& M. Malik (2000). 'Evaluation of renormalised entropy for risk stratification using heart rate variability data'. Medical and Biological Engineering and Computing 38(6):680-685.

T. Wichter, et al. (2002). 'Cardiac Autonomic Dysfunction in Brugada Syndrome'. Circulation 105(6):702-706. 
Keywords: Brugada syndrome, autonomic nervous system, heart rate variability, heart rate dynamics

\begin{abstract}
Objective: Ventricular arrhythmias in Brugada syndrome (BS) mainly occur at rest, especially during nighttime, suggesting that parasympathetic activity at night may play an important role in the arrhythmogenesis of the disease. This study examined and compared the autonomic function of symptomatic and asymptomatic BS patients overnight. Approach: We analyzed different heart rate variability (HRV) and heart rate complexity (HRC) markers in a clinical series including 87 BS patients, where 23 were symptomatic. Main results: Statistically significant differences were found in $M I R R, S D N N, S D A N N, \delta S D A N N \delta_{S D A N N}$ and SampEn, suggesting that symptomatic patients shod a-may be related to lower heart rate variability and complexity values, as well as a greater fluctuation in sinus node response to autonomic nervous system (ANS)to greater circadian fluctuations overnight. Significance: The results provide further evidence for the role of autonomic imbalance in the pathophysiology of BS, highlighting the relevance of nighttime analysis to unmask significant ANS changes. Based on these outcomes, the role of HRV and HRC assessment at night could be a step forward towards the understanding of BS and the risk for the occurrence of symptoms in these patients, with a potential future impact on therapeutic strategies.
\end{abstract}

\title{
1. Introduction
}

Brugada syndrome (BS) is a genetic disease presenting a typical electrocardiographic pattern characterized by a distinctive ST-segment elevation in right precordial leads and a high risk of sudden cardiac death (SCD) due to ventricular fibrillation (VF) in individuals with structurally normal hearts (Brugada \& Brugada 1992). 
(Priori, et al. 2013, Priori, et al. 2015). Since its first description as a distinct cardiac disease in (Brugada \& Brugada 1992), BS continues to spark a great research interest due to its high incidence, especially in Southeast Asia, and due to its association with SCD in young adults and, less frequently, in children and infants. The syndrome is estimated to be responsible for $4 \%$ of the total amount of SCD and for $20 \%$ of SCD in structurally normal hearts (Brugada, et al. 1998, Antzelevitch, et al. 2005) i

Although several markers have been reported as predictive factors of SCD occurrence, the largest series including BS patients only determined two reliable indicators of cardiac events: prior symptoms, such as recovered SCD or syncope, and spontaneous type-1 ECG pattern (Antzelevitch et al. 2005, Probst, et al. 2010). Since implantable cardioverter defibrillators (ICD) are the only effective treatment though associated with a high incidence of adverse events (Sacher, et al. 2013), while symptomatic patients must undergo an implantation, risk stratification for asymptomatic patients still remains controversial.

Ventricular arrhythmias in BS mainly occur at rest, especially at night during sleep, suggesting that parasympathetic activity may play an important role in the arrhythmogenesis of the syndrome (Matsuo, et al. 1999, Kies, et al. 2004). Indeed, some studies on cardiac autonomic nervous system (ANS) analyzed by positron emission tomography have evidenced that BS patients display some autonomic dysfunction (Kies et al. 2004, Wichter, et al. 2002, Paul, et al. 2011, Bigi, et al. 2008). Thus, changes in the autonomic modulation assessed by heart rate variations might provide useful information about the arrhythmic risk of these patients.

Previous studies assessing the autonomic function through heart rate variability (HRV) analyses along 24-hour ECG recordings have led to contradictory results (Krittayaphong, et al. 2003, Hermida, et al. 2003, Pierre, et al. 2007, Tokuyama, et al. 2014, Nakazawa, et al. 2003, Kostopoulou, et al. 2010, Behar, et al. 2016). Moreover, since linear temporal and spectral HRV parameters are unable to capture the complexity of heart rate regulation, features measuring the nonlinear dynamics of RR series, such as Sample Entropy (SampEn), could better predict the risk of suffering symptoms in BS. Although we already reported significant results in heart rate complexity (HRC) during exercise testing in a previous work including BS patients (Calvo, et al. 2017), we are not aware of any study having assessed HRC at night.

Therefore, in order to identify significant differences in the autonomic function of BS patients at different levels of risk (symptomatic and asymptomatic subjects), the aim of this study was to compare HRV and HRC markers and its circadian fluctuations at night in a clinical series including $87 \mathrm{BS}$ patients. The evaluation of heart rate variability and complexity markers may provide supplementary information that could be useful for risk stratification in asymptomatic BS patients. 


\section{Methods}

\subsection{Study population}

The 24-hour continuous ECG recordings of 87 patients diagnosed with Brugada syndrome were collected - in the context of a prospective, multicentric study conducted between 2009 and 2013 in the Cardiology department of the Rennes University Hospital, in France. Participants were enrolled in 8 French hospitals located in Rennes, Tours, Poitiers, Angers, Nantes, Bordeaux, Brest and La Rochelle. The protocol was approved by the respective local ethics committees before the study recruitment began in 2009, and all patients provided written informed consent before participation.

In accordance with the current guidelines (Priori et al. 2015, Priori et al. 2013), BS was diagnosed when a coved ST-segment elevation $(\geq 0.2 \mathrm{mV})$ was recorded in at least one right precordial lead (V1 and/or V2) located in the $2^{\text {nd }}, 3^{\text {rd }}$ or $4^{\text {th }}$ intercostal space, in the presence or absence of sodium-channel-blocking agent.

They were enrolled in 8 French hospitals located in Rennes, Tours, Poitiers, Angers, Nantes, Bordeaux, Brest and La Rochelle. The study protocel was approved by the respective local ethics committees and all participants provided written informed consent before participation.

Participant ages ranged from 19 to 79 years old $(45.63 \pm 13.03$ years old) and $71.26 \%$ were men. Based on their medical history, they were classified as symptomatic and asymptomatic. Twenty-three patients had the following documented symptoms of ventricular origin: syncope $(60.87 \%)$, cardiac arrest $(34.78 \%)$, dizziness $(13.04 \%)$ and, less frequently, palpitations $(4.35 \%)$ and nocturnal convulsions $(4.35 \%)$. The remaining 64 patients were considered as asymptomatic.

Cardioverter defibrillator implantation had been performed in 15 of $64(23.44 \%)$ asymptomatic patients, based on a positive EPS (Electrophysiological Study) test, whereas all symptomatic patients were ICD carriers. Since no relevant cardiac events were noted at night, defibrillators caused no significant effects on the recordings.

Genetic testing in search of SCN5A mutations was completed in 71 patients (21 were symptomatic) and is pending in 6 asymptomatic and 1 symptomatic subjects. The test was not performed in 9 patients (1 symptomatic), as no mutation had been identified in their families. Among 27 patients in whom the SCN5A mutation was found, 10 were symptomatic.

Table 1 summarizes the clinical characteristics of patients included in the study. Since no significant differences in age, gender and SCN5A-mutation presence between symptomatic and asymptomatic groups was noted ( $p$-vale-value $>0.05)$, similar baseline characteristics were assumed between populations.

\subsection{Signal acquisition and preprocessing}

The standard 12-lead ECG recordings of each patient were eellected and analyzed by the central board (Centre Hospitalier Universitaire de Rennes). They were acquired 
Table 1. Clinical characteristics of asymptomatic and symptomatic study groups.

\begin{tabular}{lccc}
\hline & $\begin{array}{c}\text { Asymptomatic } \\
(\mathbf{n = 6 4 )}\end{array}$ & $\begin{array}{c}\text { Symptomatic } \\
(\mathbf{n = 2 3 )}\end{array}$ & $p$-value \\
\hline Age, years old & $45.50 \pm 13.08$ & $46.00 \pm 13.16$ & 0.996 \\
Male sex, $n(\%)$ & $43(67.19 \%)$ & $19(82.61 \%)$ & 0.163 \\
ICD implantation, $n(\%)$ & $15(23.44 \%)$ & $23(100 \%)$ & - \\
Cardiac arrest, $n(\%)$ & - & $8(30.43 \%)$ & - \\
Syncope, $n(\%)$ & - & $14(60.87 \%)$ & - \\
Near syncope/dizziness, $n(\%)$ & - & $3(13.04 \%)$ & - \\
Palpitations, $n(\%)$ & - & $1(4.35 \%)$ & - \\
Nocturnal convulsions, $n(\%)$ & - & $1(4.35 \%)$ & - \\
Presence of SCN5A mutation, $n(\%)$ & $17(34.00 \%)$ & $10(47.62 \%)$ & 0.284 \\
\hline
\end{tabular}

for 24 hours, acquired with the Holter monitor (ELA medical, Sorin Group, Le Plessis Robinsson, France)and at a sampling frequency of $1000 \mathrm{~Hz}$. For this study, only the period from midnight to 6 a.m. was analyzedevaluated, so as to make sure that heart rate was being assessed autonomic analysis was performed while patients were asleep.

RR series were obtained using a noise-robust wavelet-based algorithm for QRS complex detection and subsequent R-wave peak location (Dumont, et al. 2010). So as to minimize the effect of artifacts and ectopic beats, $\mathrm{RR}$ series were filtered using a 5-beat sliding window algorithm that rejects those beats deviating more than $15 \%$ from the average length of the preceding intervals (Clifford, et al. 2002). Rejected beats were then replaced by the previous 5-beat $\mathrm{RR}$ mean (Wessel, et al. 2000), and the quality of RR series was finally verified by visual inspection.

For frequency analysis, since RR series were not uniformly sampled with respect to time, a cubic splines interpolation was applied to data, in order to obtain regularlysampled series, at a rate of $4 \mathrm{~Hz}$. Moreover, so as to remove DC and very low frequency components, RR series were high-pass filtered at $0.03 \mathrm{~Hz}$, with a $4^{\text {th }}$ order Butterworth filter applied in both forward and backward directions.

\subsection{Heart rate variability}

According to the Task Force on HRV (Camm, et al. 1996), and after performing manual verification to ensure that RR series artifacts and ectopic beats had been successfully removed, the following time-domain variables were computed: mean and standard deviation of RR intervals ( $\overline{R R}$ and $S D N N), N N 50$ as the number of pairs of adjacent RR intervals differing by more than 50 milliseconds and $p N N 50$ as the proportion of $N N 50$ divided by the total number of intervals. Additionally, MIRR was calculated as the interquartile range of the RR sequence, $S D A N N$ as the standard deviation of the entire 5-minute RR means, $S D N N 5$ min as the mean of the 5-minute RR standard deviations and $r M S S D$ as the root mean square of the successive difference between 
adjacent $R R$ intervals.

Parametric modeling analysis such as the autoregressive (AR) model using the Burg method provides high-resolution spectral estimation of short-term signals as the RR windowed segments studied in (Burg 1975). The estimation is based on the minimization of the forward and backward prediction errors using the least squares method. The AR model order was optimized for each signal, using the Broersen's combined information criterion (Broersen 2000), in order to extract the spectral HRV indices from the resulting Power Spectral Density (PSD).

The total power (TP) was obtained as the sum of the four spectral bands: ultra-low frequency (ULF: 0-0.003 Hz), very-low frequency (VLF: 0.003-0.04 Hz), low frequency (LF: 0.04-0.15 Hz) and high frequency (HF: 0.15-0.4 Hz). The HF component is defined as a marker of vagal modulation and the LF component is modulated by both the sympathetic and parasympathetic nervous systems, leading to controversial interpretations (Camm et al. 1996). These were calculated from the average of 5-minute intervals and normalized by subtracting the VLF component from the total power in order to reduce the effects of noise artifacts:

$$
\begin{aligned}
& L F_{n u}=\frac{L F}{T P-V L F} \\
& H F_{n u}=\frac{H F}{T P-V L F}
\end{aligned}
$$

The $\mathrm{LF} / \mathrm{HF}$ ratio reflects the global sympathovagal balance and can be used as a measure of this balance.

\subsection{Heart rate complexity}

The nonlinear properties of HRVwere also analyzed through the-

According to a critical review of newly developed HRV methods published after the Task Force on HRV (Sassi, et al. 2015), although several nonlinear markers, such as those capturing the chaotic behavior of HRV, have been proposed to characterize HRC, the results collected thus far are still too limited to conclude that human HRV arises from a chaotic behavior of the cardiovascular system.

Conversely, according to the same review, entropy measures such as sample entropy $(S a m p E n)$ approach, which is seem to accurately represent the responsiveness of the cardiovascular system to environmental stimuli, decreasing when RR series become more ordered and predictable. Therefore $\mathrm{HRC}$ was herein evaluated through the analysis of the entropy captured by SampEn. This marker is indeed a refined version of the traditionally used irregularity measure Approximate Entropy (ApEn) (Richman \& Moorman 2000). It is defined as the conditional probability that two sequences of $m$ consecutive data points which are similar to each other (with a tolerance $r$ ) will remain similar when one more consecutive point is included. Large values of SampEn indicate high irregularity and great complexity, whereas smaller values indicate a more regular and predictable signal, commonly associated with disease (Khandoker, 
et al. 2009, Javorka, et al. 2008, Tuzcu, et al. 2006). Based on (Lake, et al. 2002), the embedding dimension was chosen as $m=3$ and tolerance distance as

Although no consensus has been yet established for the appropriate selection of the embedding dimension $m$ and tolerance threshold $r$, based on the exhaustive evaluation performed by (Lake et al. 2002) that led to the selection of $m=3$ and $r=0.2 \cdot S D_{2}$ we chose, as in (Kim, et al. 2005. Tuzcu et al. 2006), this combination of parameters.

\subsection{Statistical analysis}

Comparisens

Kolmogorov-Smirnov tests were applied so as to assess normality on extracted markers. Since, according to the results, normal distributions could not be assumed, statistical comparisons between symptomatic and asymptomatic patients during the whole night (midnight to 6 a.m.), as well as for each interval of one hour, were evaluated by Mann-Whitney U non-parametric tests. Moreover, fluctuations in HRV at night were calculated as in (Behar et al. 2016), using a coefficient of variation $\delta=$ (maximum minimum value)/maximum value.

Logistic regression is a statistical method that finds the best fitting model to describe the relationship between a dichotomous outcome (here the presence/absence of symptoms) and a set of independent variables $X_{1}, \cdots, X_{k}$. It generates the coefficients $b_{o}, b_{1}, \cdots, b_{k}$ (and its standard errors and significance levels) that maximize the likelihood of observing the sample values in order to predict a logit transformation of the probability of suffering symptoms from the following formula:

$$
\operatorname{logit}(p)=\ln \frac{p}{1-p}=b_{0}+b_{1} X_{1}+\cdots+b_{k} X_{k}
$$

Therefore, binary logistic regression models were applied to each variable differing significantly between groups in order to predict the probability of experiencing symptoms related to those HRV and HRC measures. By calculating the odds ratios (OR) related to these models, we quantified how strongly the presence of each extracted feature may be associated with the presence of symptoms in Brugada syndrome.

HRV parameter calculations and statistical tests were performed by applying algorithms developed in our laboratory for HRV analysis, using the commercially available software MatLab (Mathworks Inc. MI, USA) and setting the level of significance at $p<0.05$.

\section{Results}

When the whole night was taken into account, similar results with no significant differences between study groups were found. However, the variation coefficient of $S D A N N, \delta S D A N N$, was significantly higher in symptomatic than in asymptomatic patients between 1 a.m. and 5 a.m. $(p<0.005)$.

Regarding heart rate variability and complexity differences analyzed for each interval of one hour, some relevant tendencies, represented by some exemplifying markers 
in Figure 1, were observed. Table 2 summarizes the mean \pm standard deviation values for symptomatic and asymptomatic patients and the associated $p$-values obtained in each significant case. Results from all the analyzed markers are provided as Supplementary material.

Table 2. Mean \pm standard deviation, for asymptomatic and symptomatic patients, and associated $p$-values of significant variables.

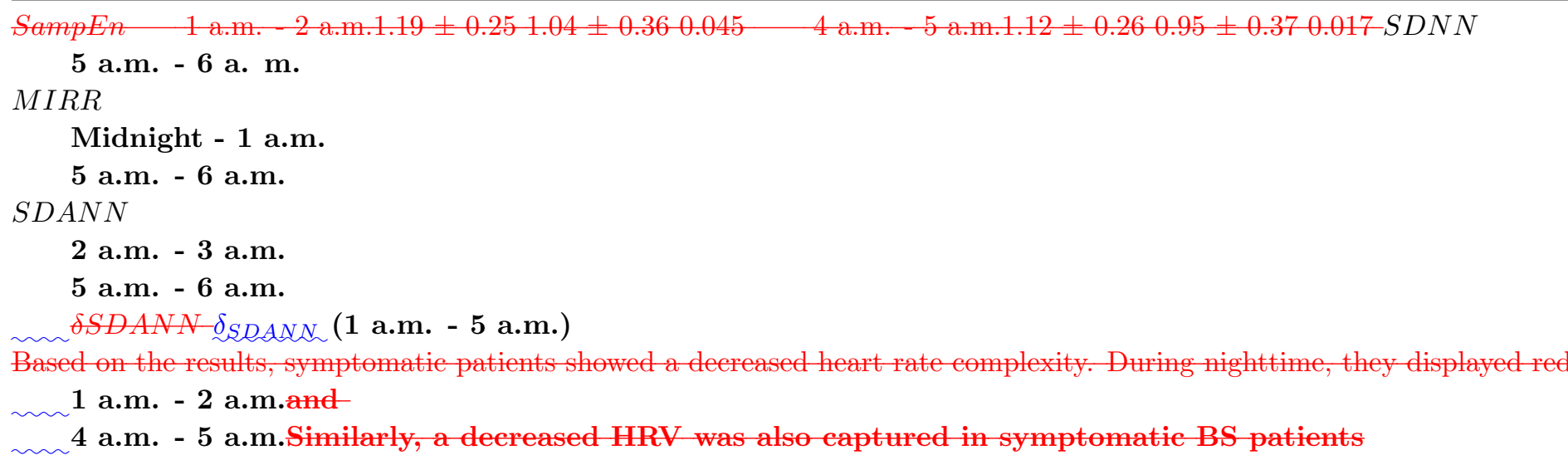

Symptomatic patients showed a decreased heart rate variability, which was statistically significant between 5 a.m. - 6 a.m., according to $S D N N, M I R R$ and $S D A N N$. Significant differences were also captured by $M I R R$ between midnight - 1 a.m. and by $S D A N N$ between 2 a.m. - 3 a.m. Similarly, they displayed reduced values for SampEn with respect to asymptomatic subjects, which turned to be significant between 1 a.m. - 2 a.m. and 4 a.m. - 5 a.m.

Table 3 summarizes the odds ratios (OR), 95\% confidence intervals (CI) and $p$ values associated to each univariate model. In summary, $M I R R$ between midnight - 1 a.m. turned to be an independent predictor for the occurrence of symptoms in univariate analysis with a 1.7\% decreased risk per one point increase. Between 2 a.m. - 3 a.m., $S D A N N$ displayed a decreased risk of the 3.1\%. $\delta S D A N N$ between 1 a.m. - 5 a.m. was also a significant predictor of symptoms, as well as SampEn between 1 a.m. - 2 a.m. and between 4 a.m. - 5 a.m. showed a decreased risk of the $82.9 \%$ and $87.1 \%$, respectively, per one point of SampEn increase.

Figure 1 represents the circadian variation of $\mathrm{HRV}$ and $\mathrm{HRC}$ at night for both groups of patients, by showing the evolution of the mean, and the $25 \%$ of the standard deviation, at each hour for the most significant autonomic markers. Likewise, the main tendency of SampEn and MIRR is represented by fitting linear regression lines for each group of patients. The graphs show a similar evolution along the night in terms 
Table 3. Odds ratios, $95 \%$ confidence intervals and $p$-values from univariate analysis of significant autonomic markers.

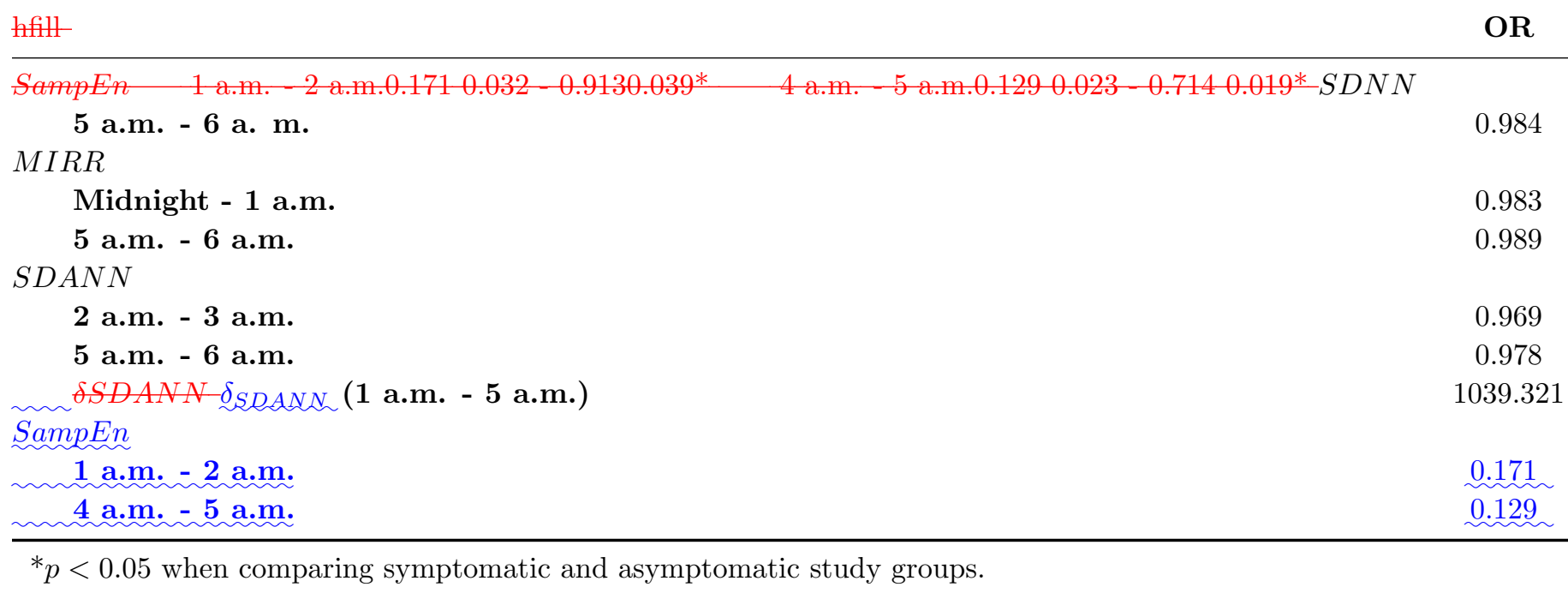

${ }^{*} p<0.05$ when comparing symptomatic and asymptomatic study groups.

of regression line slopes between both groups, but symptomatic patients display lower mean heart rate variability and complexity values.

Figure 1. Mean and $25 \%$ of standard deviation, for $S a m p E n$ and mean $M I R R$ for on symptomatic and asymptomatic BS patients at each hour during nighttime $\left({ }^{*} p<0.05\right)$. Dotted black lines are fitted linear regression lines that represent the main tendencies of each variable along the night, for both study groups.


\section{Discussion}

In this study, the autonomic function of 87 BS patients was analyzed at night through several HRV and HRC markers. Although some previous studies have already assessed 
HRV on these patients, this manuscript presents a more extensive clinical series than those reported thus far and proposes the first comparison of HRC between symptomatic and asymptomatic BS patients overnight.

In a clinical series of 27 patients with BS, of whom 10 were asymptomatic subjects with Brugada ECG, and 45 controls, Krittayaphong et al (Krittayaphong et al. 2003) found that symptomatic BS patients showed lower HRV and lower vagal tone at night compared to controls, as well as lower diurnal and higher overnight heart rates compared to asymptomatic subjects and controls. Similarly, Hermida et al (Hermida et al. 2003) detected a significant reduction in $S D A N N$ at night when comparing Holter recordings in 21 symptomatic and 26 asymptomatic BS patients. Pierre et al (Pierre et al. 2007) also confirmed in a clinical series of $46 \mathrm{BS}$ patients and 46 controls that HRV in BS was significantly lower with respect to healthy subjects. Tokuyama et al (Tokuyama et al. 2014) results showed a significant reduction of the LF and HF components in BS patients with previous VF when analyzing a series of 12 symptomatic (with previous VF), 17 asymptomatic and 16 healthy individuals. The results showed a decreased circadian variation of the autonomic response over 24 hours, with respect to controls. Conversely, Nakazawa et al (Nakazawa et al. 2003) analyzed, in 24-hour ECG recordings, the autonomic function of $27 \mathrm{BS}$ patients (10 of them had a history of VF and 17 did not) and of 26 healthy subjects. They found higher vagal and reduced sympathetic activities (increase of HF component and decrease of $\mathrm{LF} / \mathrm{HF}$ ) in BS patients with previous VF compared to controls, as well as an increase of HF component when compared to asymptomatic BS patients. Kostopoulou et al (Kostopoulou et al. 2010) did not find any significant differences in HRV between 20 patients with BS and 20 age-matched controls, although a high susceptibility to vasovagal syncope during head-up tilt test in BS patients was noticed.

In our study, according to time-domain HRV markers, symptomatic patients showed lower MIRR, SDNN and $S D A N N$ values, mainly during the last hour (5 a.m. - 6 a.m.). Therefore, patients with higher risk for cardiac events seem to show a decreased HRV, following the tendency published in previous works (Krittayaphong et al. 2003, Hermida et al. 2003, Pierre et al. 2007, Tokuyama et al. 2014), emphasizing differences during the last hour of sleep.

From HRC analysis, statistically significant results were observed between 2 a.m. - 3 a.m. and 4 a.m. - 5 a.m. Symptomatic patients showed more regularity in RR series, which might be related to a worse cardiac health condition since a reduced complexity in HR has been associated with disease (Khandoker et al. 2009, Javorka et al. 2008, Tuzcu et al. 2006). The ANS is controlled by complex interactions resulting from feedback loops of nonlinear systems that permit the organism to adapt to particular physiological conditions, such as metabolic variations, stress or disease. Thus, according to previous studies, a reduction in the complexity of biological control systems reflects a decreased organism adaptability, generally associated with age and disease (Goldberger, et al. 2002, Rabinovich \& Abarbanel 1998, Magrans, et al. 2010, Smith, et al. 2005).

In a previous study, we reported reduced values of HRC in symptomatic BS 
patients with respect to asymptomatic subjects, during recovery after physical stress testing (Calvo et al. 2017). As it was suggested, this affected ANS dynamics when parasympathetic activity is predominant confirms previous studies that highlight the importance of autonomic imbalance in the genesis of life-threatening arrhythmias in BS (Matsuo et al. 1999, Kies et al. 2004). Indeed, ventricular arrhythmias and sudden cardiac death (SCD) mainly occur at rest and especially during sleep, when vagal tone is dominant.

Regarding circadian fluctuations, based on $\delta S D A N N \delta_{S D A N N}$ results, we observed significantly greater variations during nighttime in symptomatic than in asymptomatic patients, which concurs with previous studies having compared these groups during daytime and when the night was analyzed between midnight and 4 a.m. Our results eonfirm an increased sinus node response to ANS in patients having experienced symptoms. As suggested by (Behar et al. 2016), this increase in sinus node $\delta_{\text {SDANN }}$ might be caused by wider fluctuations in the autonomic tone, or by a greater sinus node autonomic receptors sensitivity. Therefore, the risk of arrhythmias might be incremented by these greater variations in sinus node response to $\mathrm{ANS}$.

Then, we included those statistically significant variables in univariate logistic regression models in order to estimate the discrimination power of each parameter. According to HRV results, MIRR turned to be an independent predictor between midnight and 1 a.m.; $S D A N N$ at 2 a.m. - 3 a.m; and $\delta S D A N N-\delta_{S D A N D}$ between 1 a.m. and 5 a.m. HRC captured by SampEn was also a significant predictor for the occurrence of cardiac events.

Thus, our findings provide further evidence for the role of autonomic imbalance in the pathophysiology of the Brugada syndrome. Although we did not find significant differences between symptomatic and asymptomatic groups in HRV markers characterizing the HF component that would directly relate the loss of cardiac variability and complexity with an increase in parasympathetic activity, the results highlight the relevance of night analysis in order to unmask significant autonomic changes that could not be captured at daytime, during a regular ECG examinationin symptomatic BS patients.

These results support the idea that decreased nocturnal HR variability and complexity, as well as a greater circadian variation, could be related to a greater risk of suffering symptoms in Brugada syndrome. Measuring HRV not only by conventional but also by nonlinear methods could be a helpful tool to better understand autonomic alterations in BS, with a potential impact on risk stratification and, thus, on therapeutic strategies.

Although cardioverter defibrillators are the only proven effective treatment thus far, since they are associated with a high incidence of adverse events, the decision to implant them on asymptomatic subjects is still contentious, even if they represent around the $60 \%$ of diagnosed patients. Thus, the proposed indices are presented as a potential instrument to better identify those asymptomatic BS patients at high risk who may benefit from an ICD implantation. Moreover, they might also be applied to data 
acquired from ICDs on already implanted BS patients, in order to control their risk of suffering symptoms during follow-up.

\section{Conclusions}

In this study, we analyzed and compared several HRV and HRC markers in 87 subjects in order to identify autonomic differences between symptomatic and asymptomatic BS patients at night. Statistically significant results were obtained for differences were observed in several temporal $(M I R R, p=0.014 ; S D N N, \delta S D A N N, p=0.029$; $\delta_{\text {SDANA }} p<0.005 ; S D A N N$ and,$\left.p=0.025\right)$ and nonlinear (SampEn, $p=0.017$ ) markers, especially during the last period of sleep. Symptomatic patients showed The results suggest that symptomatic patients may be related to lower heart rate variability and complexity, and no significant differences in sympathetic and parasympathetic tones were noted between groups. Moreover, greater fluctuations in the sinus node response to the ANS was observed in symptomatic patientsvalues, as well as to greater circadian fluctuations overnight.

However, the-The study presents some limitations that should be noted. First, the clinical influence of the analyzed markers can only be proved if a direct relationship between heart rate alterations and cardiac events is identified. Here, symptomatic patients had documented symptoms but since no ventricular arrhythmias were induced during the recordings, heart rate variations in symptomatic patients cannot be directly associated to a greater risk for suffering symptoms. Moreover, the analysis is based on a relatively small population of 87 patients and, thus, conclusions on predictors of symptoms cannot be extracted. Finally, although differences in the autonomic function of different sleep stages have been reported, since accurate sleep-phase identifications require electroencephalogram, electrooculogram and electromyogram data not available for this study the obtained results may not be a reliable measure of the autonomic function at night.

Nevertheless, the observed differences between symptomatic and asymptomatic BS patients indicate important trends of clinical relevance that could provide new insights in the understanding of the physiopathology of the syndrome. Future work will be based on combining these parameters, together with other relevant markers of risk, on a multivariate classification method capable of identifying BS patients who may benefit from an ICD implantation.

\section{Acknowledgments}

This work was supported by the French Ministry of Health (Programme Hospitalier de Recherche Clinique - PHRC Regional). MC thanks la Caixa Foundation (Spain) and DR acknowledges Lefoulon-Delalande Foundation (France) for financial support. 


\section{References}

C. Antzelevitch, et al. (2005). 'Brugada Syndrome: Report of the Second Consensus Conference: Endorsed by the Heart Rhythm Society and the European Heart Rhythm Association'. Circulation 111(5):659-670.

N. Behar, B. Petit, V. Probst, F. Sacher, G. Kervio, J. Mansourati, P. Bru, A. Hernandez \& P. Mabo (2016). 'Heart rate variability and repolarization characteristics in symptomatic and asymptomatic Brugada syndrome'. Europace p. euw224.

M. A. B. Bigi, A. Aslani \& A. Aslani (2008). 'Significance of cardiac autonomic neuropathy in risk stratification of Brugada syndrome'. Europace 10(7):821-824.

P. Broersen (2000). 'Finite sample criteria for autoregressive order selection'. Signal Processing, IEEE Transactions on $\mathbf{4 8}(12): 3550-3558$.

J. Brugada, R. Brugada \& P. Brugada (1998). 'Right Bundle-Branch Block and ST-Segment Elevation in Leads V1 Through V3 : A Marker for Sudden Death in Patients Without Demonstrable Structural Heart Disease'. Circulation 97(5):457-460.

P. Brugada \& J. Brugada (1992). 'Right bundle branch block, persistent $\{\mathrm{ST}\}$ segment elevation and sudden cardiac death: A distinct clinical and electrocardiographic syndrome: A multicenter report'. Journal of the American College of Cardiology 20(6):1391 - 1396.

J. P. Burg (1975). Maximum Entropy Spectral Analysis. Phd thesis, Stanford University, Stanford, CA 94305.

M. Calvo, P. Gomis, D. Romero, V. Le Rolle, N. Béhar, P. Mabo \& A. Hernández (2017). 'Heart rate complexity analysis in Brugada syndrome during physical stress testing'. Physiological measurement 38(2):387.

A. Camm, et al. (1996). 'Heart rate variability: standards of measurement, physiological interpretation and clinical use. Task Force of the European Society of Cardiology and the North American Society of Pacing and Electrophysiology'. Circulation 93(5):1043-1065.

G. Clifford, P. McSharry \& L. Tarassenko (2002). 'Characterizing artefact in the normal human 24hour RR time series to aid identification and artificial replication of circadian variations in human beat to beat heart rate using a simple threshold'. In Computers in Cardiology, 2002, pp. 129-132. IEEE.

J. Dumont, A. Hernandez \& G. Carrault (2010). 'Improving ECG Beats Delineation With an Evolutionary Optimization Process'. Biomedical Engineering, IEEE Transactions on 57(3):607615.

A. L. Goldberger, C.-K. Peng \& L. A. Lipsitz (2002). 'What is physiologic complexity and how does it change with aging and disease?'. Neurobiology of aging 23(1):23-26.

J.-S. Hermida, A. Leenhardt, B. Cauchemez, I. Denjoy, G. Jarry, F. Mizon, P. Milliez, J.-L. Rey, P. Beaufils \& P. Coumel (2003). 'Decreased nocturnal standard deviation of averaged NN intervals'. European heart journal 24(22):2061-2069.

M. Javorka, Z. Trunkvalterova, I. Tonhajzerova, J. Javorkova, K. Javorka \& M. Baumert (2008). 'Short-term heart rate complexity is reduced in patients with type 1 diabetes mellitus'. Clinical Neurophysiology 119(5):1071-1081.

A. H. Khandoker, H. F. Jelinek \& M. Palaniswami (2009). 'Identifying diabetic patients with cardiac autonomic neuropathy by heart rate complexity analysis'. Biomedical engineering online 8(1):3.

P. Kies, et al. (2004). 'Abnormal Myocardial Presynaptic Norepinephrine Recycling in Patients With Brugada Syndrome'. Circulation 110(19):3017-3022.

W.-S. Kim, Y.-Z. Yoon, J.-H. Bae \& K.-S. Soh (2005). 'Nonlinear characteristics of heart rate time series: influence of three recumbent positions in patients with mild or severe coronary artery disease'. Physiological measurement 26(4):517.

A. Kostopoulou, et al. (2010). 'Disorders of the Autonomic Nervous System in Patients With Brugada Syndrome: A Pilot Study'. Journal of Cardiovascular Electrophysiology 21(7):773-780.

R. Krittayaphong, G. Veerakul, K. Nademanee \& C. a. Kangkagate (2003). 'Heart rate variability in 
patients with Brugada syndrome in Thailand'. European Heart Journal 24(19):1771-1778.

D. E. Lake, J. S. Richman, M. P. Griffin \& J. R. Moorman (2002). 'Sample entropy analysis of neonatal heart rate variability'. American Journal of Physiology-Regulatory, Integrative and Comparative Physiology 283(3):R789-R797.

R. Magrans, P. Gomis, P. Caminal \& G. Wagner (2010). 'Multifractal and nonlinear assessment of autonomous nervous system response during transient myocardial ischaemia'. Physiological measurement 31(4):565-580.

K. Matsuo, T. Kurita, M. Inagaki, M. Kakishita, N. Aihara, W. Shimizu, A. Taguchi, K. Suyama, S. Kamakura \& K. a. Shimomura (1999). 'The circadian pattern of the development of ventricular fibrillation in patients with Brugada syndrome'. European Heart Journal 20(6):465-470.

K. Nakazawa, T. Sakurai, A. Takagi, R. Kishi, K. Osada, T. Nanke, F. Miyake, N. Matsumoto \& S. Kobayashi (2003). 'Autonomic imbalance as a property of symptomatic Brugada syndrome'. Circulation journal : official journal of the Japanese Circulation Society 67(6):511-514.

M. Paul, M. Meyborg, P. Boknik, U. Gergs, W. Schmitz, G. Breithardt, T. Wichter \& N. Joachim (2011). 'Autonomic Dysfunction in Patients with Brugada Syndrome: Further Biochemical Evidence of Altered Signaling Pathways'. Pacing and Clinical Electrophysiology 34(9):1147-1153.

B. Pierre, D. Babuty, P. Poret, C. Giraudeau, O. Marie, P. Cosnay \& L. Fauchier (2007). 'Abnormal nocturnal heart rate variability and QT dynamics in patients with Brugada syndrome'. Pacing and clinical electrophysiology 30(s1):S188-S191.

S. G. Priori, et al. (2015). '2015 ESC Guidelines for the management of patients with ventricular arrhythmias and the prevention of sudden cardiac death: The Task Force for the Management of Patients with Ventricular Arrhythmias and the Prevention of Sudden Cardiac Death of the European Society of Cardiology (ESC) Endorsed by: Association for European Paediatric and Congenital Cardiology (AEPC)'. European heart journal 36(41):2793-2867.

S. G. Priori, et al. (2013). 'Executive summary: HRS/EHRA/APHRS expert consensus statement on the diagnosis and management of patients with inherited primary arrhythmia syndromes'. Europace 15(10):1389-1406.

V. Probst, et al. (2010). 'Long-term prognosis of patients diagnosed with Brugada syndrome results from the FINGER Brugada Syndrome Registry'. Circulation 121(5):635-643.

M. Rabinovich \& H. Abarbanel (1998). 'The role of chaos in neural systems'. Neuroscience 87(1):5-14.

J. S. Richman \& J. R. Moorman (2000). 'Physiological time-series analysis using approximate entropy and sample entropy'. American Journal of Physiology-Heart and Circulatory Physiology $\mathbf{2 7 8}(6): \mathrm{H} 2039-\mathrm{H} 2049$.

F. Sacher, et al. (2013). 'Outcome after implantation of a cardioverter-defibrillator in patients with Brugada syndrome'. Circulation 128(16):1739-1747.

R. Sassi, et al. (2015). 'Advances in heart rate variability signal analysis: joint position statement by the e-Cardiology ESC Working Group and the European Heart Rhythm Association co-endorsed by the Asia Pacific Heart Rhythm Society'. EP Europace 17(9):1341-1353.

R. G. Smith, L. Betancourt \& Y. Sun (2005). 'Molecular endocrinology and physiology of the aging central nervous system'. Endocrine reviews 26(2):203-250.

T. Tokuyama, et al. (2014). 'Deterioration of the circadian variation of heart rate variability in Brugada syndrome may contribute to the pathogenesis of ventricular fibrillation'. Journal of cardiology 64(2):133-138.

V. Tuzcu, S. Nas, T. Börklü \& A. Ugur (2006). 'Decrease in the heart rate complexity prior to the onset of atrial fibrillation'. Europace 8(6):398-402.

N. Wessel, A. Voss, J. Kurths, A. Schirdewan, K. Hnatkova \& M. Malik (2000). 'Evaluation of renormalised entropy for risk stratification using heart rate variability data'. Medical and Biological Engineering and Computing 38(6):680-685.

T. Wichter, et al. (2002). 'Cardiac Autonomic Dysfunction in Brugada Syndrome'. Circulation 105(6):702-706. 